\title{
Interplay between polarity and chirality in the electric-field-responsive columnar phase of a dipeptide derivative
}

\author{
Naomi Koizumi ${ }^{1}$, Govindaswamy Shanker ${ }^{2}$, Fumito Araoka ${ }^{1}$, Ken Ishikawa ${ }^{1}$, \\ Channabasaveshwar V Yelamaggad ${ }^{2}$ and Hideo Takezoe ${ }^{1}$
}

We studied unconventional polar switching associated with an electro-optical response in the columnar oblique phase of a dipeptide derivative. Slow and careful cooling allows us to obtain a large monodomain with the column axis perpendicular to the substrates. The cross-section of the columns is birefringent, and the principal axis rotates about the column axis by applying an electric field parallel to the column axis, yielding the following characteristics: (1) the rotation angle exhibits linear dependence on an applied electric field; (2) the rotational sense is reversed by reversing the field direction; and (3) the optical isomers exhibit opposite rotational sense. A second-harmonic generation (SHG) signal was observed only under an electric field. We observed a dependence of the SHG intensity and infrared (IR) absorption on the incidence angle. Asymmetric and symmetric variations were observed when the sample was rotated about the slow and fast in-plane optical axes, respectively. From these experimental results, we proposed a model of molecular packing in the columnar phase; the molecules stack with their molecular planes tilted from the column axis, and the polar order is cancelled within the neighboring columns. NPG Asia Materials (2012) 4, e11; doi:10.1038/am.2012.20; published online 13 April 2012

Keywords: chirality; columnar liquid crystal; columnar oblique phase; dipeptide; infrared absorption; polar switching; second-harmonic generation

\section{INTRODUCTION}

Some biomolecules and their derivatives exhibit liquid crystalline properties. These materials have attracted much attention because they are one of the keys to understanding self-organization in biological systems. In addition, they are interesting from a materials science viewpoint, particularly for synthesizing new biomimetic materials. Hydrogen bonding is important for forming superstructures in organic systems because it is much stronger than van der Waals interactions. ${ }^{1}$ Hydrogen bonding is polar, thus sequential hydrogen bonds make a column polar. ${ }^{2-7}$ If the direction of the hydrogen bonds can be reversed by an external electric field, the column could become ferroelectric. Even without reversing the hydrogen-bond direction, hydrogen-bonded chains may exhibit interesting polar properties.

Recently, Yelamaggad et al. ${ }^{8}$ synthesized unique discotic columnar (Col) liquid crystals composed of dipeptide derivatives. Some of the derivatives exhibited a single polarization reversal current peak during application of a triangular wave voltage. These derivatives also showed an electro-optical (EO) response. From these observations, the researchers concluded that the phase is ferroelectric. They proposed a model of molecular alignment in a column in which molecules align in a helical manner. However, they did not quantitatively analyze the polarization direction or the relationship between polarization and optical switching.

To examine the detailed nature of the EO response and polar switching of the dipeptide derivatives, we carried out highly sensitive electro-optic, second-harmonic generation (SHG), and polarized IR absorption measurements using monodomain samples. We discovered new EO switching: that is, a rotation about the axis of the columns, which have an optical anisotropy in their cross-sectional surfaces. We also discovered an asymmetric incidence-angle dependence of the SHG signals and the IR absorption, which is inconsistent with the proposed model of a helical molecular arrangement along the column axis. ${ }^{8}$ We propose a new model based on the experimental observations described in this work.

\section{EXPERIMENTAL PROCEDURES}

The sample used was a D (L)-dipeptide derivative. The molecular structure and phase sequence of the compound are shown in Figure 1. The sample was introduced into sandwich-type cells using two $\mathrm{SrF}_{2}$ or indium-tin-oxidecoated glass plates via capillary action. The cell surfaces were coated with polyimide (JSR AL1254) for electrical insulation. No rubbing was made.

${ }^{1}$ Department of Organic and Polymeric Materials, Tokyo Institute of Technology, Meguro-ku, Tokyo, Japan and ${ }^{2}$ Centre for Soft Matter Research, Bangalore, India Correspondence: Professor H Takezoe, Department of Organic and Polymeric Materials, Tokyo Institute of Technology, 2-12-1 0-okayama, Meguro-ku, Tokyo 152-8552, Japan. E-mail: takezoe.h.aa@m.titech.ac.jp

Received 29 December 2011; revised 22 February 2012; accepted 23 February 2012 
The optical textures were obtained using a polarizing microscope (Optiphot-POL, Nikon, Tokyo, Japan) equipped with a hot stage and a controller (FP82HT \& FP90, Mettler-Toledo AG, Switzerland). Polarization reversal current measurements were performed under triangular and modified square waveform voltages using sample cells with a patterned electrode $\left(4 \times 5 \mathrm{~mm}^{2}\right)$. The polarization reversal current was recorded using a digital storage oscilloscope (TDS220, Tektronix, Inc., Beaverton, OR, USA).

The rotation angle of the optical axis under an electric field was precisely measured by detecting the transmittance change as described below. A sample cell was set between crossed polarizers; the in-plane optical axis was set at $22.5^{\circ}$ from the polarizer axis. With this arrangement, the intensity of the transmitted light linearly depends on the rotational angle of the in-plane optical axis. ${ }^{1}$ A sinusoidal wave field was applied perpendicular to the substrates, and the modulation in the transmitted intensity was measured with a lock-in amplifier (5610B, NF corporation, Yokohama, Kanawaga, Japan) to determine the rotational angle. Accuracy of this measurement is two orders of magnitude higher than that of the direct measurement of the rotational angle.

To examine the polar structure, SHG measurements were carried out using a femtosecond Ti:S laser (Vitesse 800 , wavelength $=800 \mathrm{~nm}$, repetition $=80$ $\mathrm{MHz}$, pulse duration $=100 \mathrm{fs}$, Coherent Inc., Santa Clara, CA, USA) as a fundamental light source. SH light was detected by a photomultiplier tube (H7421-40, Hamamatsu Photonics, Hamamatsu, Shizuoka, Japan) through the transmission direction. Neither focusing lenses nor collecting lenses were used. The signals were fed into a photon counter and sent to a personal computer.

Fourier transform infrared (FTIR) absorption spectra were obtained using an FTIR spectrometer (Winspec50, JEOL Ltd., Akishima, Tokyo, Japan). The

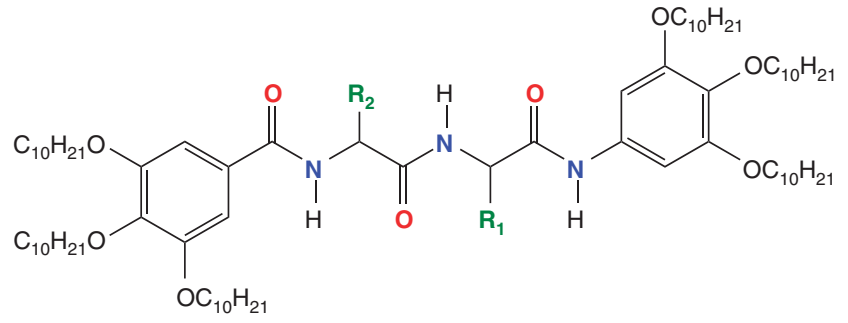

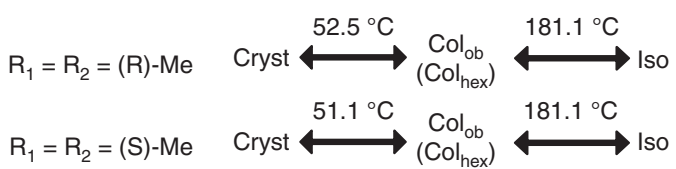

Figure 1 Molecular structure and phase sequence of the $D(L)$-dipeptide derivative. cell was composed of $\mathrm{SrF}_{2}$ substrates, and the cell thickness was $2.5 \mu \mathrm{m}$. The sample was kept at $150^{\circ} \mathrm{C}$ during the measurements.

X-ray diffraction was measured using a Rigaku RU200 (Rigaku Corporation, Akishima, Tokyo Japan) $\mathrm{X}$-ray source $(\mathrm{Cu} \mathrm{K} \alpha$ line, $12 \mathrm{~kW})$ equipped with a temperature control unit.

\section{RESULTS}

\section{Monodomain growth and polymorphism}

The observations made using a polarizing microscope indicate that upon slowly cooling the cell from the isotropic phase, nucleation of a single domain produced a star-like dendritic shape (Figure 2a, left). Further cooling led to a monodomain cell (Figure $2 \mathrm{a}$, middle) with a typical size exceeding $1 \mathrm{~cm}^{2}$. The dendritic domain was obliquely distorted in shape and had six branches of varying interbranching angles (Figure 2a, left). The cell exhibited some brightness under crossed polarizers. From these observations, this domain was deemed the oblique columnar $\left(\mathrm{Col}_{\mathrm{ob}}\right)$ phase, which was consistent with the $\mathrm{X}$-ray observation discussed below. The column axis spontaneously aligned perpendicular to the substrate surface, so we could observe the system along the column axis. Application of an electric field caused a brightness change (Figure 2a, middle-to-right). Minor domains that could be assigned to the columnar hexagonal $\left(\mathrm{Col}_{\text {hex }}\right)$ phase also emerged with a six-fold symmetry and were darker (Figure 2b, left) than the $\mathrm{Col}_{\mathrm{ob}}$ phase. Neither texture nor brightness changed immediately after the application of an electric field. However, an irreversible change to a smooth and brighter texture occurred after prolonged field application (Figure 2b, middle-to-right and Supplementary Figure S1, bottom-to-center). This change can be attributed to the electric-field-induced phase transition from $\mathrm{Col}_{\text {hex }}$ to $\mathrm{Col}_{\mathrm{ob}}$. The melting points of these two phases were nearly identical. We found that the two columnar phases can stably coexist depending upon the experimental conditions, such as cooling and heating rates and the application of an electric field (see Supplementary Figure S1).

X-ray diffraction confirmed that these two domains were different columnar phases (Supplementary Figures S2 and S3). The obliquely distorted phase had small birefringence, which is consistent with $\mathrm{Col}_{\mathrm{ob}}$ (see Supplementary Figure S3). The other phase exhibited a dendritic structure with six branches and an interbranching angle of approximately $60^{\circ}$, which is characteristic of a hexagonal lattice. An X-ray diffraction pattern of this phase at $80^{\circ} \mathrm{C}$ showed diffraction spots peaking at $3.64^{\circ}$ and $6.24^{\circ}$ with a reciprocal $d$-spacing ratio of $\sqrt{ } 3: 1$ corresponding to $(10)$ and (11) reflections, respectively, which is

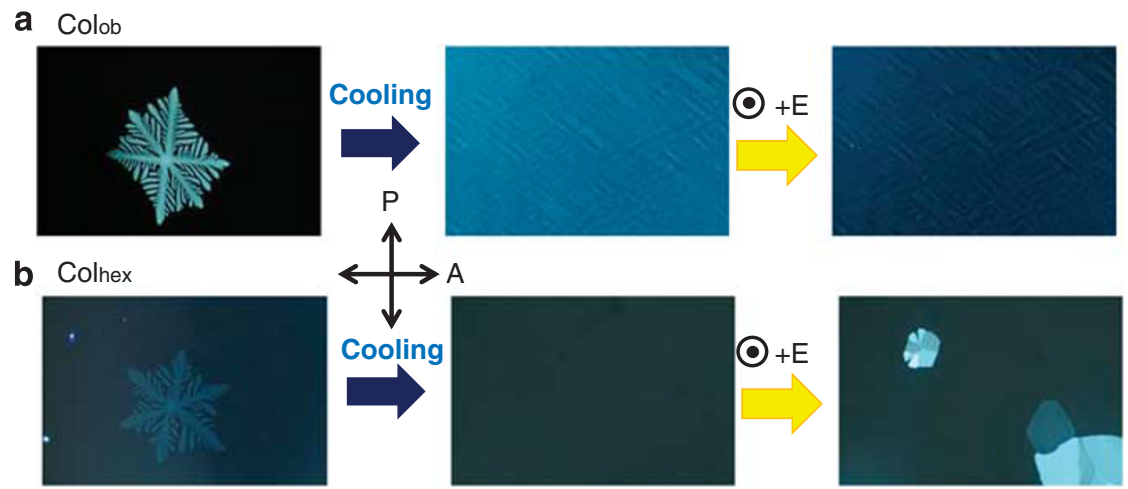

Figure 2 Polarizing microscope textures of the $\mathrm{Col}_{\mathrm{ob}}$ and $\mathrm{Col}_{\text {hex }}$ phases. (a) Growth of a monodomain having a distorted oblique shape in the Col $\mathrm{ob}$ phase,

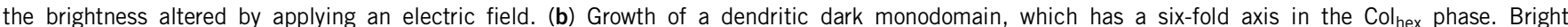
domains emerged with the application of an electric field. 
a pattern characteristic of $\mathrm{Col}_{\text {hex }}$ (see Supplementary Figure S2). Viewing under crossed polarizers produced darkness, which is coincident with the hexagonal packing of the columns nearly perpendicular to the substrates. In this paper, we focus only on the $\mathrm{Col}_{\mathrm{ob}}$ phase in which the columns orient perpendicular to the substrates.

\section{Electroclinic-like switching}

We observed a slight change in the brightness with the application of an electric field in $\mathrm{Col}_{\mathrm{ob}}$. To clarify the origin of and measure this small brightness change, we set the in-plane optical axis at $22.5^{\circ}$ from the polarizer axis. By applying a positive (upward) voltage to the D-dipeptide compound, the brightness decreased slightly; however, it increased when a negative (downward) voltage was applied. These changes confirm that the brightness change originates in the optical axis rotation in which the rotational sense is opposite under the positive and negative fields. We also confirmed that the L-dipeptide compound exhibited the opposite rotation under the same experimental conditions. The difference in the behaviors of the D- and L-dipeptides and the opposite rotations with the field application of the opposite senses indicate an interplay between chirality and polarity. Most of the following experiments were carried out using the D-dipeptide compound.

We measured the rotational angle as a function of the applied electric field at $4 \mathrm{~Hz}$ (see Supplementary Information for details). As shown in Figure 3a, the angle increases almost linearly with increasing field and saturates at approximately $22 \mathrm{~V}_{\mathrm{pp}} \mu \mathrm{m}^{-1}$, with a saturation
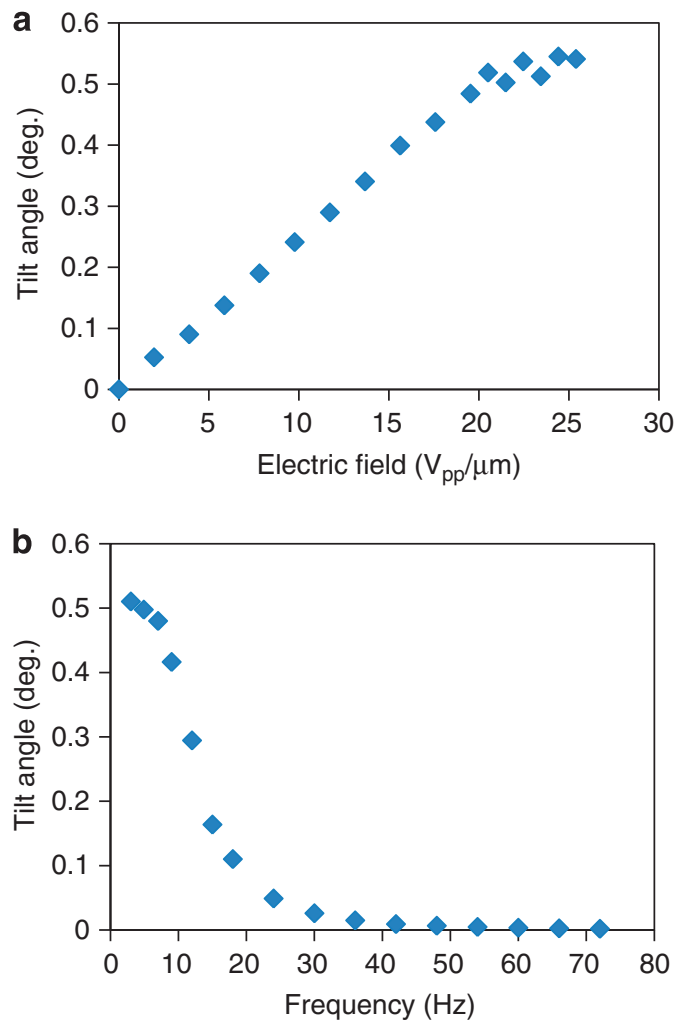

Figure 3 Emergence of a tilt angle by applying an electric field. (a) The field dependence of the in-plane optical axis rotation at $150{ }^{\circ} \mathrm{C}$. The angle linearly increases with the field and saturates at approximately $0.54^{\circ}$ and $22 \mathrm{~V}_{\mathrm{pp}} \mu \mathrm{m}^{-1}$. (b) The frequency dependence of the angle at $150{ }^{\circ} \mathrm{C}$ under an electric field of $15.6 \mathrm{~V}_{\mathrm{pp}} \mu \mathrm{m}^{-1}$. The response is on the order of $10 \mathrm{~Hz}$. value of $0.54^{\circ}$. Similar EO rotation was observed in some columnar phases. $^{9-11}$ The behavior appears similar to the electroclinic (EC) effect observed in the smectic A phase composed of chiral molecules $\left(\mathrm{SmA}^{\star}\right){ }^{12,13}$ The optical axis rotation is proportional to the electric field strength, $E$, in the EC effect as a result of a linear coupling of the applied electric field and an induced polarization. Therefore, the tilt is reversed by reversing $E$ or by using an opposite enantiomer.

The EO response in $\mathrm{Col}_{\mathrm{ob}}$ is qualitatively similar to other $\mathrm{EC}$ (in $\mathrm{SmA}^{*}$ ) and EO (in a chiral columnar phase) rotation behaviors, but it is otherwise unique. The rotation axis of $\mathrm{Col}_{\mathrm{ob}}$ is along the column axis, not perpendicular to the column axis as in the other reported columnar phases. ${ }^{9-11}$ In addition, there are notable differences in the rotation angle and the response time: the rotation angle in the $\mathrm{Col}_{\mathrm{ob}}$ phase is small (less than $1^{\circ}$ ), whereas the saturation values were greater than $10^{\circ}$ in other phases. ${ }^{9-11}$ Moreover, the relaxation frequency was approximately $12 \mathrm{~Hz}$ (Figure $3 \mathrm{~b}$ ) in the $\mathrm{Col}_{\mathrm{ob}}$ phase, which is much lower than that in the $\mathrm{SmA}^{*}$ phase.

\section{Polar properties}

We measured the SHG to examine the polar order and its relationship to the EO response. Figure 4 shows the electric field dependence of the SH light intensity at an oblique incidence. The SHG signal was only observed under an electric field, suggesting that this material is apolar in the absence of an electric field. This result is inconsistent with observations made previously by Yelamaggad et al., ${ }^{8}$ who observed a single reversal current peak, which is characteristic of a ferroelectric liquid crystal. The SHG intensity increases continuously and saturates at nearly the same voltage, $25 \mathrm{~V}_{\mathrm{PP}} \mu \mathrm{m}^{-1}$, as the EO response, meaning that the polar order is closely related to the rotation of the optical axis.

If the $\mathrm{Col}_{\mathrm{ob}}$ phase is a ferroelectric phase, the finite polarization field should remain in the absence of an electric field, contrary to the present findings. To explore the possibility of a ferroelectric phase in detail, we performed a reversal current measurement using a largesized monodomain sample. Figure $5 \mathrm{a}$ shows the reversal current behavior upon application of a reverse-square pulsed voltage. The total polarization ratio (in areas of reversed current) associated with the onset, reversal, and termination of the field was approximately 4:10:3, which was close enough to $1: 2: 1$, if arbitrariness in drawing the appropriate baselines was taken into account. This result indicates

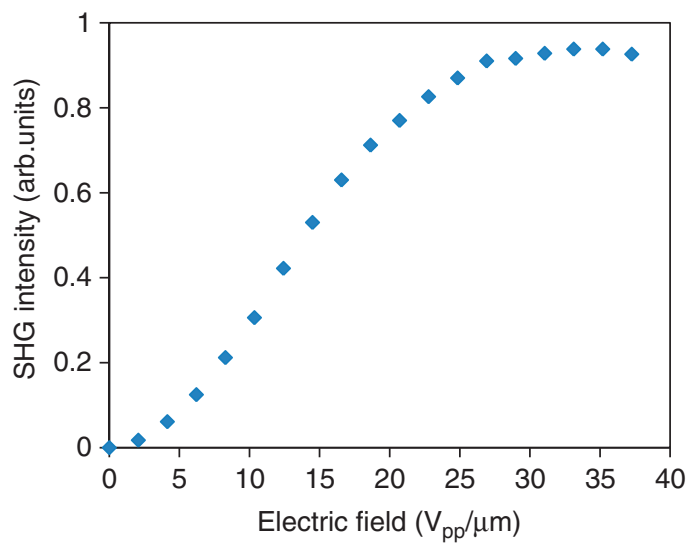

Figure $4 \mathrm{SHG}$ intensity as a function of a field at an oblique incidence of $45^{\circ}$ with the $\mathrm{p}_{\mathrm{in}}-\mathrm{p}_{\text {out }}$ polarization combination at $150^{\circ} \mathrm{C}$. The intensity saturates at approximately $25 \mathrm{~V}_{\mathrm{pp}} \mu \mathrm{m}^{-1}$, which is close to the saturation field strength for the tilt angle. 

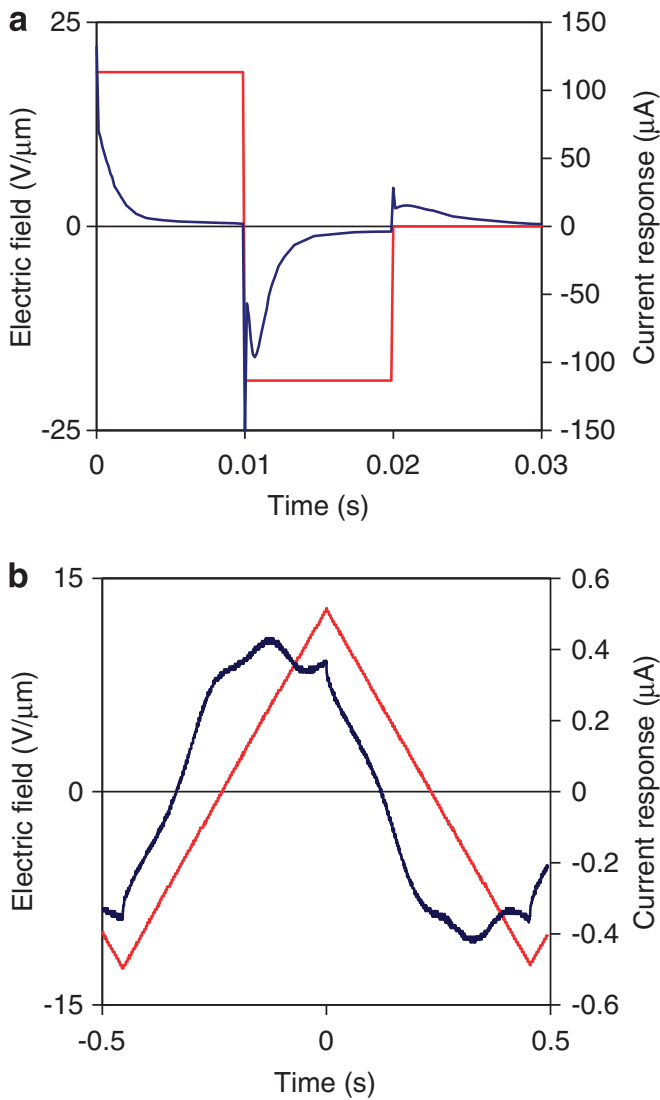

Figure 5 The switching current profile under the application of (a) a rectangular reversed pulse and (b) a triangular wave field of $1.1 \mathrm{~Hz}$ at $150^{\circ} \mathrm{C}$.

that polarization is induced by a field, but the polar order cannot remain and is relaxed to an apolar orientation once the field is removed. Next, we applied a triangular wave voltage of $1.2 \mathrm{~Hz}$ as shown in Figure 5b. Double reversal current peaks characteristic of antiferroelectric liquid crystals ${ }^{14}$ were clearly observed. Spontaneous polarization was obtained at approximately $360 \mathrm{nC} \mathrm{cm}^{-2}$ from both the deconvoluted first and second peaks. The difference between the present (antiferroelectric behavior) and the previous (ferroelectric behavior) results may stem from the different domain sizes and the frequency applied. For these measurements, we used a single domain sample of greater than $1 \mathrm{~cm}^{2}$, which is much larger than the electrode area $\left(0.2 \mathrm{~cm}^{2}\right)$. We observed a single peak profile when using a multidomain cell with smaller domain sizes or frequencies higher than $2.3 \mathrm{~Hz}$. The same result was achieved with a monodomain cell at frequencies higher than $2.3 \mathrm{~Hz}$ (Supplementary Figure S4).

\section{Angle dependence of the SHG signal and IR absorption}

We found that an SHG signal was observed even at normal incidence under an electric field. To determine the in-plane direction of the SHG polarization, that is, the projection of the nonlinear polarization onto the plane perpendicular to the column axis, the azimuthal angle dependence of the SHG signal was measured. Figure 6 shows the SHG intensity plotted against the angle between the parallel polarizer axes and the in-plane optical fast axis at normal (open symbols) and slightly oblique $\left(\sim 2^{\circ}\right.$, closed symbols) incidences. The optical slow and fast axes were experimentally determined using a sensitive tint plate. The in-plane slow axis can be taken parallel to the molecular

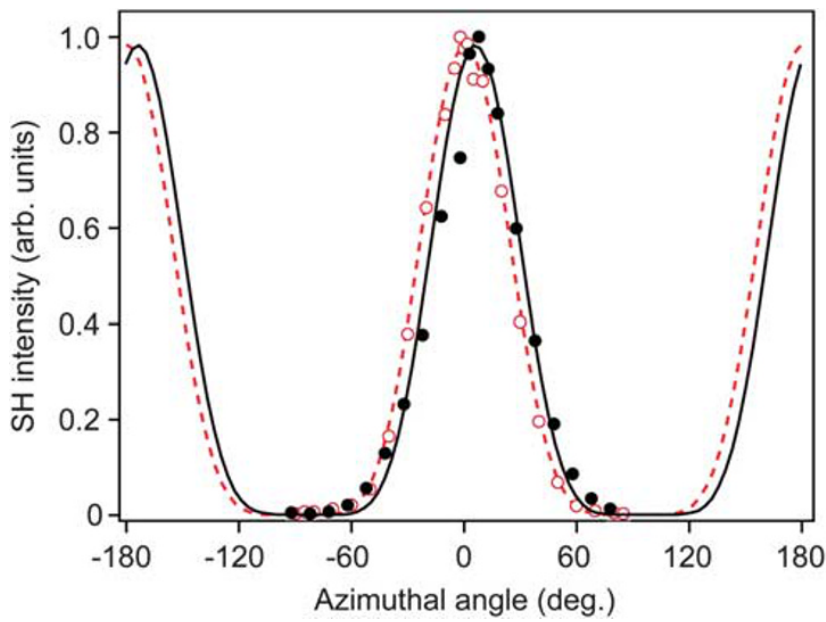

Figure 6 SHG intensity plotted against the angle between the paralle polarizers and the slow axis at a normal (solid symbols) and a slightly oblique incidence (open symbols). The maximum SHG intensity is obtained for the polarizer directions parallel to the fast axis for normal incidence and is slightly deviated from the fast axis. Solid and broken curves are simultaneous best fits to both data sets. The parameters, the nonlinear electric susceptibility components, obtained by the fitting are shown in the text, indicating the importance of the chiral contribution. In fact, the nonzero chiral components are the origin of the SHG peak deviation from the fast axis for oblique incidence.

long axis that connects two phenyl rings. This result is reasonable because the refractive index for light polarized parallel to this direction must be larger than that for the other axis. The angle dependence for normal incidence indicates that the maximum SHG intensity occurs at approximately $0^{\circ}$, whereas the maximum slightly deviates from $0^{\circ}$ for oblique incidence. Hence, the projection of the nonlinear polarization axis is nearly parallel to the in-plane fast axis. The assignment of the fast and slow axes is consistent with the obtained direction of the nonlinear polarization and should include the $\mathrm{C}=\mathrm{O}$ and $\mathrm{N}-\mathrm{H}$ components, which are nearly perpendicular (the optical fast axis) to the molecular axis (the optical slow axis). Simultaneous best fits for both data sets were successfully made (as illustrated by the dotted and solid curves in Figure 6) using nonlinear susceptibility components: $\chi_{\mathrm{xxx}}=0.549, \chi_{\mathrm{xyy}}=\chi_{\mathrm{yxy}}=0.049$, and $\chi_{\mathrm{xyz}}=\chi_{\mathrm{yzx}}=\chi_{\mathrm{zxy}}=1$ in which the $\mathrm{x}$-axis is parallel to the substrate and incidence planes, and the $\mathrm{z}$-axis is the column axis (normal to the substrate). Fitting can be made with zero $\chi_{\text {zzz }}$ because of the nearly normal incidence condition. Quite a large contribution of chiral components is necessary and is consistent with the present chiral system.

Existence of an SHG signal in the normal incidence condition indicates that the nonlinear polarization is not parallel to the column axis, although the electric field is applied parallel to that axis. To obtain detailed information regarding the nonlinear polarization direction, the SHG intensity was measured as a function of the incidence angle using a polarization combination of $\mathrm{p}$-in and p-out. The results in which a sample cell was rotated about the in-plane slow axis (closed symbols) and fast axis (open symbols) are shown in Figure 7. For the rotation about the in-plane slow axis, the incidenceangle dependence is asymmetric about the normal incidence, as shown in Figure 7, which shows a peak (approximately $25^{\circ}$ ) and a minimum $\left(-14^{\circ}\right)$ at the same angles irrespective of the applied electric field strength $\left(6.4 \mathrm{~V} \mathrm{~mm}^{-1}\right.$ and $\left.10.3 \mathrm{~V} \mu \mathrm{m}^{-1}\right)$. In contrast, when the sample was rotated about the in-plane fast axis, the 


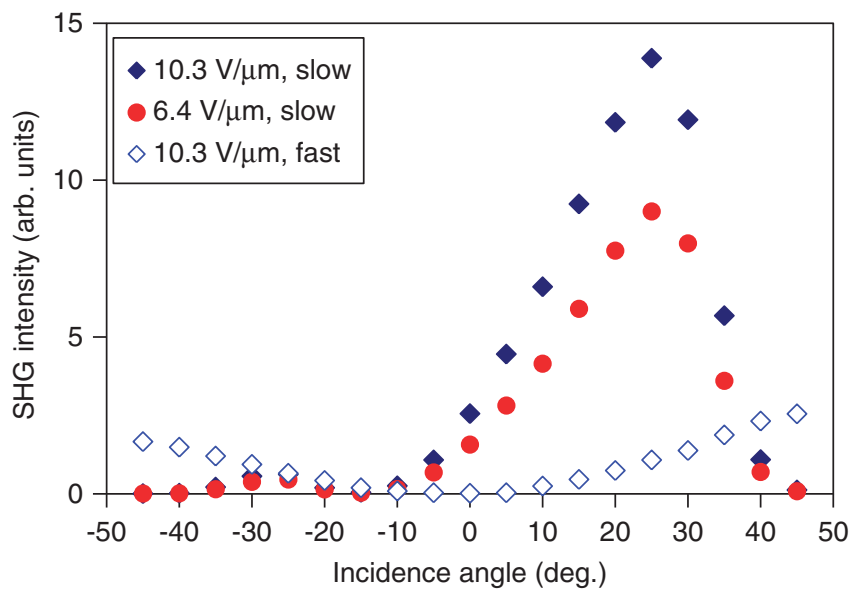

Figure 7 Incidence-angle dependence of SHG intensity. The change in the incidence angle was made by the rotation about the in-plane optical slow (closed) and fast (open) axes. The angle showing the maximum SHG intensity is independent of the applied field strength.

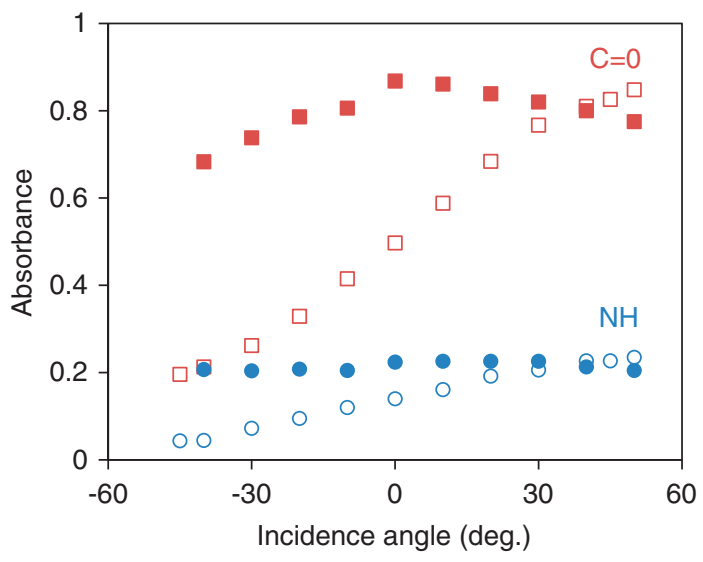

Figure 8 Incidence-angle dependence of IR absorption for the $\mathrm{C}=\mathrm{O}$ (red) and $\mathrm{N}-\mathrm{H}$ (blue) groups. Asymmetric behavior is observed when rotating about the optical slow axis (closed symbols). Rotation about the optical fast axis shows symmetrical dependence (open symbols).

incidence-angle dependence was nearly symmetrical with the normal incidence. Thus, the nonlinear polarization is found to slightly deviate $\left(\sim 9^{\circ}\right)$ from the column direction toward the fast axis. This estimation was made with the assumption that the refractive index of the sample is 1.6 given that the SHG intensity becomes zero when the incident fundamental beam propagates along the direction of nonlinear polarization.

We measured the IR absorption spectra to obtain details on the orientation of the molecules within the columns (see Supplementary Figures S5 and S6). The incidence-angle dependencies of the IR peak intensity of $\mathrm{C}=\mathrm{O}$ and $\mathrm{N}-\mathrm{H}$ stretching at 1630 and $3280 \mathrm{~cm}^{-1}$ are shown in Figure 8. As with the SHG measurements, when the sample was rotated about the in-plane slow axis, the incidence-angle dependence was asymmetric about the normal incidence (closed symbols). This asymmetric angular dependence suggests that the $\mathrm{C}=\mathrm{O}$ and $\mathrm{N}-\mathrm{H}$ bond directions occur at finite angles to the surface. The rotation to positive (negative) angles turns the bonds so that they become perpendicular (parallel) to the IR ray axis, and the absorbance increases (decreases). In contrast, the incidence-angle dependence is
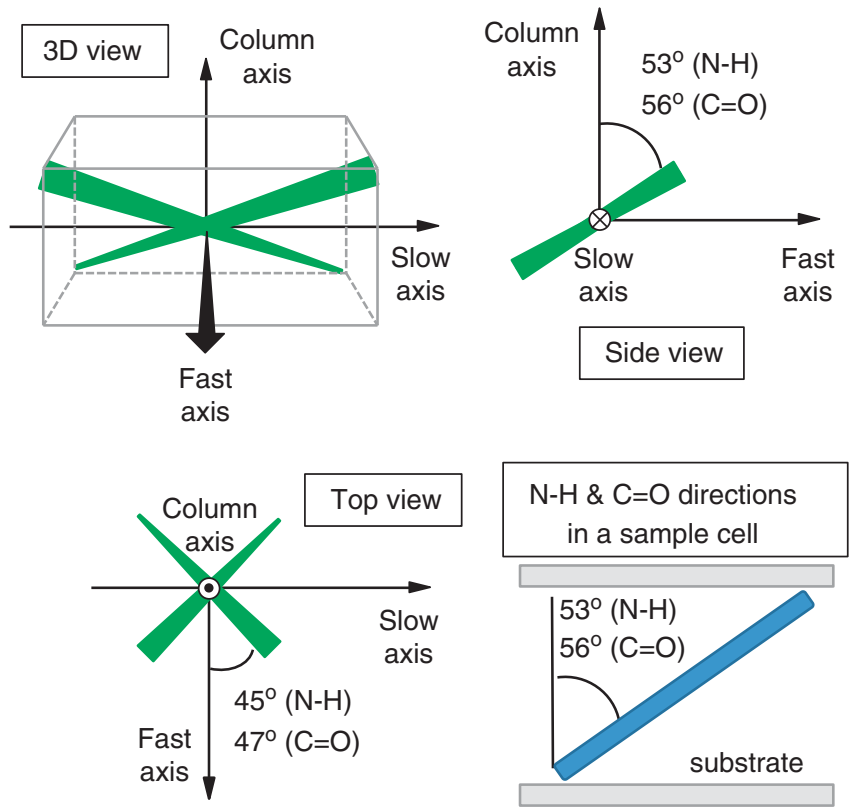

Figure 9 Schematic illustration of the direction of the $\mathrm{C}=\mathrm{O}$ and $\mathrm{N}-\mathrm{H}$ bonds. The thick end of green bars represents the upward direction and the thin end represents the downward direction with respect to the sheet. The obtained angles for the $\mathrm{C}=\mathrm{O}$ and $\mathrm{N}-\mathrm{H}$ groups are indicated in the figure.

symmetrical (open symbols) when the sample is rotated about the in-plane fast axis. The absorbance exhibited a broad maximum at the normal incidence and was nearly constant over the rotation to both senses for the $\mathrm{C}=\mathrm{O}$ and $\mathrm{N}-\mathrm{H}$ bonds, respectively. We evaluated the directions of the $\mathrm{C}=\mathrm{O}$ and $\mathrm{N}-\mathrm{H}$ bonds by fitting the angle dependences about the in-plane fast and slow axes. The results are schematically illustrated in Figure 9, which aids in understanding the qualitative feature of the incidence-angle dependence on the IR absorbance about the fast and slow axes. This assignment is qualitatively consistent with the above-mentioned IR absorption results. The $\mathrm{C}=\mathrm{O}$ bond is tilted from the column axis by approximately $56^{\circ}$, with a spread of $\pm 47^{\circ}$ from the in-plane fast axis. The angles are $53^{\circ}$ and $45^{\circ}$ for the $\mathrm{N}-\mathrm{H}$ bond, respectively.

\section{DISCUSSION}

We now discuss the molecular orientation in the $\mathrm{Col}_{\mathrm{ob}}$ phase based on the above information, as shown in Figure 9. The electric field dependence of the SHG signal and the reversal current measurements show that the $\mathrm{Col}_{\mathrm{ob}}$ phase is macroscopically apolar in the absence of an electric field. Yelamaggad et al. ${ }^{8}$ suggested the formation of a helical structure along the column axis. From the in-plane anisotropy of the IR absorption and the SHG intensity, however, it is obvious that the molecules do not form a helix but stack with their long axes and molecular planes nearly parallel to one another in each column. The most appropriate molecular arrangement for the formation of hydrogen bonds is shown in Figure 10a (right) in which the neighboring molecules are mutually rotated by $\pi$ about the molecular plane normal. Without this rotation, parallel molecular packing via hydrogen bonding is impossible. If the neighboring molecular planes are parallel to one another, all dipole moments are cancelled out. However, if we assume a slight alternate tilt of the molecular plane (Figure 10a, left), hydrogen bonding directed toward one of the $\mathrm{O}$ atoms (arrows) is favored (dotted line), resulting in a macroscopic polarization in each column. This situation is plausible because of the 

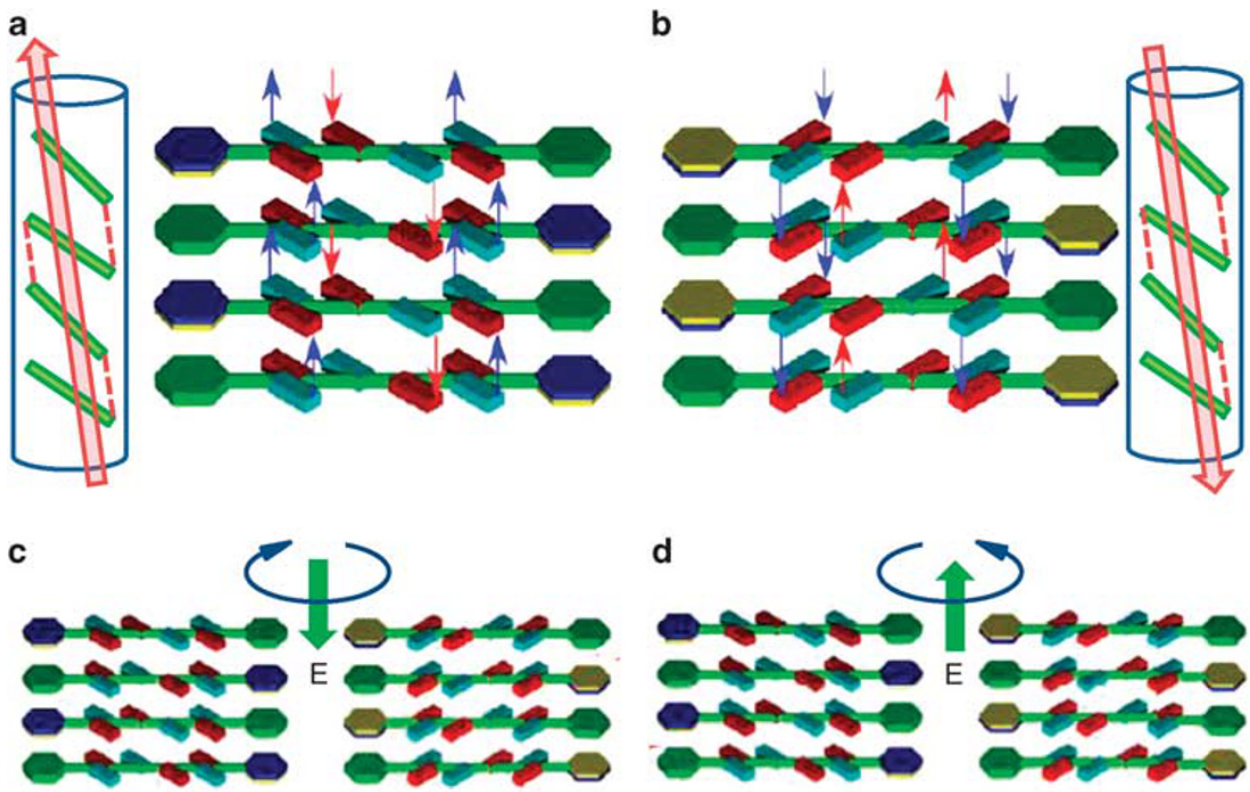

Figure 10 Molecular arrangements in the two neighboring columns and the rotation created by the application of an electric field. (a) Molecular packing model in the $\mathrm{Col}_{\mathrm{ob}}$ phase. The red and blue blocks represent the $\mathrm{C}=\mathrm{O}$ and $\mathrm{N}-\mathrm{H}$ groups, respectively. One of the phenyl rings is colored dark blue and yellow to distinguish the left/right and up/down of an asymmetric molecule. The hydrogen bonds, $\mathrm{H} \ldots \mathrm{O}$ are represented by blue and red arrows for the major and minor polarization directions, respectively. The molecular planes are not exactly parallel in neighboring molecules, as shown at left, so that the $\mathrm{H} \ldots \mathrm{O}$ directions are uniquely determined. (b) Molecular packing in the neighboring column. The directions of the blue and red streams are opposite in the two columns so that no macroscopic polarization remains in the entire system. (c) Electric field response of the columns. Molecules rotate about the column axis by applying an electric field along the column axis. (d) The rotational sense is made opposite by reversing the field.

steric repulsion of the methyl groups in the neighboring molecules. Note that the number of upward blue arrows is larger than that of the downward red arrows in Figure 10a. A slightly tilted nonlinear polarization to the fast axis from the column was obtained experimentally and is consistent with the model. Thus, the macroscopic apolar structure is possible only when the number of columns with up (Figure 10a) polarizations equals the number with down (Figure 10b) polarizations. Both a periodic variation and a random arrangement in the up and down dipoles are possible without fluctuation in the $\mathrm{Col}_{\mathrm{ob}}$ phase.

Next, we discuss the electric field response (Figures 10c and d) and should consider the macroscopic polarizations, which are antiparallel to one another in neighboring columns. By applying an electric field parallel to the column axis, a torque is exerted to tilt the column. Because the directional senses of the induced tilt are opposite in neighboring columns, this action does not easily occur. An additional rotational torque is exerted on the column about the column axis due to coupling between the polarization $P$ and $E$ given the chirality of the molecular system. When $E$ is reversed or compounds with different optical isomers are used, an opposite rotation occurs. A nonlinear polarization $P^{\mathrm{NL}}$ is also induced by $E$ and is in the plane that includes the fast and column axes, which is consistent with the experimental results illustrated in Figures 6 and 7.

We can qualitatively explain the effect using the same argument used in the EC effect of the $\mathrm{SmA}^{*}$ phase. ${ }^{12}$ If an electric field $E$ is applied parallel to the column axis, a macroscopic polarization $P$ is induced. In a nonchiral system, the plane containing the optical fast axis exists in a plane perpendicular to the column axis, and $P$ is a mirror plane. However, when the chirality breaks the mirror symmetry, the free energy for the rotation of the optical axis is no longer symmetric. In this way, EC-like coupling, which is proportional to $\theta P$ in the free energy describing the present system, leads to a linear dependence of $\theta$ and $E$. However, the microscopic origin of the EC-like effect is difficult to discuss. In fact, we measured the FTIR spectra under an electric field to see whether any changes occur to the molecular orientations or their particular groups. The reorientation of the functional groups could not be detected by applying an electric field because the effect is small. Highly sensitive IR measurements using modulation spectroscopy are indispensable for analyzing the detailed mechanism of this effect.

In summary, novel antiferroelectric properties were studied using a monodomain of the $\mathrm{Col}_{\mathrm{ob}}$ phase of a peptide derivative. The directions of the $\mathrm{C}=\mathrm{O}$ and $\mathrm{N}-\mathrm{H}$ bonds were determined using the characteristic incidence-angle dependence of the FTIR absorptions. The system became SHG-active and showed characteristic incidenceangle dependence only under an electric field. A possible model for the molecular arrangement in the $\mathrm{Col}_{\mathrm{ob}}$ phase was proposed based on the SHG and FTIR measurements; molecules stack in an antiparallel fashion in a single column, and any macroscopic polarization is cancelled out by an antiferroelectric ordering of the columns. We observed an electric-field-induced SHG and a rotation of the optical axis about the column axis, both of which saturated at the same electric field strength. This unique experimental observation indicates an electroclinic-like effect in the chiral system:- that is, a coupling between the induced polarization and the rotation angle. The rotational sense is opposite with the use of an opposite field direction or different optical isomers.

\section{ACKNOWLEDGEMENTS}

This work was partly supported by Grants-in Aid for Scnience Research (B) (23310072) by the Ministry of Education, Science, Sports and Culture. 
1 Desiraju, G. R. The $\mathrm{C}-\mathrm{H} \ldots \mathrm{O}$ hydrogen bond: structural implications and supramolecular design. Acc. Chem. Res. 29, 441-449 (1996).

2 Kishikawa, K. Nakahara, S., Nishikawa, Y., Kohmoto, S. \& Yamamoto, M. A ferroelectrically switchable columnar liquid crystal phase with achiral molecules: superstructures and properties of liquid crystalline ureas. J. Am. Chem. Soc. 127, 2565-2571 (2005)

3 Takezoe, H., Kishikawa, K. \& Gorecka, E. Switchable columnar phases. J. Mater. Chem. 16, 2412-2416 (2006).

4 Okada, Y., Matsumoto, S., Araoka, F., Goto, M., Takanishi, Y., Ishikawa, K., Nakahara, S., Kishikawa, K. \& Takezoe, H. Three relaxation processes from an electric-fieldinduced polar structure in a columnar liquid crystalline urea derivative. Phys. Rev E. 76, 041701-1-6 (2007).

5 Okada, Y., Matsumoto, S., Takanishi, Y., Ishikawa, K., Nakahara, S., Kishikawa, K. \& Takezoe, H. Polarization switching in a columnar liquid crystalline urea as studied by optical second-harmonic generation interferometry. Phys. Rev. E 72, 020701(R)-1-4 (2005)

6 Miyajima, D., Araoka, F., Takezoe, H., Kim, J., Kato, K., Takata, M. \& Aida, T. Columnar liquid crystal with a spontaneous polarization along the columnar axis. J. Am. Chem. Soc. 132, 8530-8531 (2010).

7 Miyajima, D., Araoka, F., Takezoe, H., Kim, J., Kato, K., Takata, M. \& Aida, T. Electricfield-responsive handle for large-area orientation of discotic columnar liquid crystalline molecules in millimeter-thick films. Angew. Chem. Int. Ed. 50, 7865-7869 (2011).
8 Yelamaggad, C. V., Shanker, G., Rao, R. V. R., Rao, D. S. S., Prasad, S. K. \& Babu, V. V. S. Supramolecular helical fluid columns from self-assembly of homomeric dipeptides. Chem. Eur. J. 14, 10462-10471 (2008).

9 Scherowsky, G. \& Chen, X. H. New switchable columnar liquid-crystals. Liq. Cryst. 17, 803-810 (1994).

10 Bock, H. \& Helfrich, W. Field dependent switching angle of a columnar pyrene. Liq. Cryst. 18, 707-713 (1995).

11 Heppke, G., Kruerke, D., Lohning, C., Lotzsch, D., Moro, D., Muller, M. \& Sawade, H. New chiral discotic triphenylene derivatives exhibiting a cholesteric blue phase and a ferroelectrically switchable columnar mesophase. J. Mater. Chem. 10, 2657-2661 (2000).

12 Garoff, S. \& Meyer, R. B. Electroclinic effect at ac phase-change in a chiral smectic liquid-crystal. Phys. Rev. Lett. 38, 848-851 (1977).

13 Qiu, R., Ho, J. T. \& Hark, S. K. Electroclinic effect above the smectic-A-smectic-C* transition. Phys. Rev. A 38, 1653-1655 (1988).

14 Cross, L.E. Relaxor ferroelectrics. Ferroelectrics 76, 241-267 (1987)

(c)

OMERIEHISRESERV

This work is licensed under the Creative Commons

Attribution-NonCommercial-No Derivative Works 3.0

Unported License. To view a copy of this license, visit http:// creativecommons.org/licenses/by-nc-nd/3.0/

Supplementary Information accompanies the paper on the NPG Asia Materials website (http://www.nature.com/am) 